https://helda.helsinki.fi

\title{
Predictive role of free prostate-specific antigen in a prospective active surveillance program (PRIAS)
}

\section{Vasarainen, Hanna}

$2015-11$

Vasarainen, H, Salman , J , Salminen , H , Valdagni , R , Pickles , T , Bangma , C , Roobol , M J \& Rannikko , A 2015 , ' Predictive role of free prostate-specific antigen in a prospective active surveillance program (PRIAS) ' , World Journal of Urology , vol. 33 , no. 11, pp.

1735-1740 . https://doi.org/10.1007/s00345-015-1542-3

http://hdl.handle.net/10138/158377

https://doi.org/10.1007/s00345-015-1542-3

publishedVersion

Downloaded from Helda, University of Helsinki institutional repository.

This is an electronic reprint of the original article.

This reprint may differ from the original in pagination and typographic detail.

Please cite the original version. 


\title{
Predictive role of free prostate-specific antigen in a prospective active surveillance program (PRIAS)
}

\author{
Hanna Vasarainen ${ }^{1} \cdot$ Jolanda Salman $^{2} \cdot$ Heidi Salminen $^{1} \cdot$ Riccardo Valdagni $^{3} \cdot$ \\ Tom Pickles ${ }^{4} \cdot$ Chris Bangma $^{2} \cdot$ Monique J. Roobol $^{2} \cdot$ Antti Rannikko $^{1}$
}

Received: 23 November 2014 / Accepted: 19 March 2015 / Published online: 31 March 2015

(C) Springer-Verlag Berlin Heidelberg 2015

\begin{abstract}
Purpose To evaluate the utility of percentage of free serum PSA (\%fPSA) as a predictor of adverse rebiopsy findings, treatment change and radical prostatectomy (RP) findings in a prospective active surveillance (AS) trial. Methods Patients enrolled in the global PRIAS study with baseline \%fPSA available were included. Putative baseline predictors (e.g. PSA, \% fPSA) of adverse rebiopsy findings were explored using logistic regression analysis. Association of variables with treatment change and RP findings over time were evaluated with Cox regression analysis. Active treatment-free survival was assessed with a KaplanMeier method.

Results Of 3701 patients recruited to PRIAS, 939 had $\%$ fPSA measured at study entry. Four hundred and thirtyeight of them had \%fPSA available after 1 year. Median follow-up was 17.2 months. First rebiopsy results were available for 595 patients and of those, $144(24.2 \%)$ had adverse findings. A total of $283(30.1 \%)$ patients discontinued surveillance, of those $181(64.0 \%)$ due to protocolbased reasons. Although median \%fPSA values were significantly lower in patients who changed treatment, according
\end{abstract}

Hanna Vasarainen

hanna.vasarainen@hus.fi

1 Department of Urology, Peijas Hospital, Helsinki University Central Hospital and University of Helsinki, P.O. Box 900, 00029 Helsinki, Finland

2 Department of Urology, Erasmus Medical Center, Rotterdam, The Netherlands

3 Department of Radiation Oncology 1, Fondazione IRCCS Istituto Nazionale dei Tumori, Milan, Italy

4 Department of Radiation Oncology and Developmental Radiotherapeutics, University of British Columbia, and BC Cancer Agency, Vancouver, Canada to the multivariate regression analysis, initial \%fPSA value was not predictive for treatment change or adverse rebiopsy findings. However, the probability of discontinuing AS was significantly lower in patients with "favourable" initial $\%$ fPSA characteristics and \%fPSA during follow-up (initial \%fPSA $\geq 15$ and positive \%fPSA velocity) compared to those with "adverse" \%fPSA characteristics (initial \%fPSA $<15$ and negative \%fPSA velocity).

Conclusions Diagnostic \%fPSA provides no additional prognostic value when compared to other predictors already in use in AS protocols. However, \%fPSA velocity during surveillance may aid in predicting the probability for future treatment change.

Keywords Prostate cancer - Active surveillance . Prostate-specific antigen $\cdot$ Free prostate-specific antigen

\section{Introduction}

With PSA testing being part of daily clinical practice, the issue of overdiagnosis and overtreatment of prostate cancer (PC) becomes more relevant. The number of likely overdiagnosed low-risk cancers has increased dramatically and constitutes about one half of the newly diagnosed cases [1]. Active surveillance (AS) is a management option to consider in men with a diagnosis of localized low-risk PC instead of immediate curative treatment [2].

The aim of AS is to prevent or postpone treatment of a low-risk PC to alleviate side effects inherent to all contemporary radical PC treatments. The challenge is not only to correctly classify cancers to low-risk category, but also to be able to find PCs, that were initially miss-classified or are likely to progress, in a timely fashion. Current AS protocols utilize tools such as serial PSA measurements, digital rectal 
examination (DRE) and rebiopsies, but these clearly lack performance as demonstrated by the relatively high proportion (24-46\%) of patients referred to active therapy [3-7]. Therefore, new tools such as multiparametric MRI, MRIultrasound fusion $[8,9]$ and novel biomarkers are topic of ongoing urologic research [10]. Multiparametric MRI and MRI-ultrasound fusion were also added to the PRIAS study program in 2014.

Previous studies have shown that \%fPSA (free/total PSA ratio in percentages) in serum has predictive value both at diagnosis and with radical prostatectomy (RP) outcome [11-13]. Also, PSA measurements over time (PSA kinetics) have shown predictive power beyond a single PSA measurement in providing a more dynamic picture of PC activity and tumour behaviour [14]. Here, we hypothesize that \%fPSA has a predictive role in AS. Specifically, we want to address whether \% PPSA value at diagnosis or during follow-up associates with adverse rebiopsy or RP findings and whether treatment change can be predicted.

\section{Patients and methods}

Study patients participated in the international prospective AS study named Prostate Cancer research International: Active Surveillance (PRIAS) [15]. PRIAS inclusion criteria are $\mathrm{PC}$ diagnosis, PSA $\leq 10 \mathrm{ng} / \mathrm{mL}$, tumour(T)-stage $\leq 2$, PSA density (PSA-D) $<0.2 \mathrm{ng} / \mathrm{mL} / \mathrm{cc}$, maximum two positive biopsies and Gleason score $\leq 6(3+3)$. A candidate for AS should also be fit for radical treatment. Patients are monitored carefully; PSA is measured every 3 months and DRE is performed every 6 months during the first 2 years. After 2 years, PSA is measured every 6 months and DRE is performed yearly. Repeat biopsies are taken 1, 4 and 7 years after inclusion. Biopsies are taken under TRUS guidance, and a prostate volume-dependent number of biopsy cores is advised. PSA doubling time (PSA-DT) is calculated yearly, and if it is 3-10 years, rebiopsy is recommended. Criteria for the advice to switch to active treatment are PSA-DT $<3$ years, clinical T-stage $>2, \mathrm{PC}$ in more than two biopsy cores and/or Gleason score $>6(3+3)$.

fPSA measurement is not mandatory as a part of the PRIAS protocol, but in some participating centres, it was routinely measured. \%fPSA values measured at study entry and at 1 year were available for statistical analysis. The RP data used in analyses were available from the Finnish arm of the PRIAS study.

PSA density (PSA-D) was calculated as total PSA (ng/ $\mathrm{mL}$ ) divided by prostate volume (cc) at diagnosis. Clinical stage T2 was not divided into subgroups due to the low number of cases. PSA-DT was divided into three categories: PSA-DT negative/>10 years, PSA-DT 3-10 years and PSA-DT $<3$ years. \%fPSA velocity was the difference between \%fPSA values at 1 year and diagnosis. For sub analyses, the population was stratified into four categories: (1) diagnostic \% fPSA $\geq 15$ and positive \%fPSA velocity, (2) Diagnostic \%fPSA $\geq 15$ and negative \%fPSA velocity, (3) diagnostic \%fPSA $<15$ and positive velocity and (4) $\%$ fPSA $<15$ and negative velocity. The cut-off value 15 (\%fPSA) was chosen, as being widely used in clinical practice.

Reclassification in rebiopsy was defined as Gleason sum $>6(3+3)$ and/or $\geq 3$ PC positive biopsy cores. For this analysis, only the results of the first rebiopsy were used due to the small number of patients with both \%fPSA and second/third rebiopsy result available. Pathological stage T3 and/or Gleason sum $>6(3+3)$ were considered adverse RP findings. Time to active treatment was calculated from the study inclusion to the date of discontinuation of AS. For analyses, only discontinuations due to protocol-based reasons were included.

The Statistical Package for the Social Sciences (SPSS, version 19.0; IBM) was used for the data analysis. The cutoff level of statistical significance was set at $p<0.05$ in all tests. The $p$ values were calculated using the Mann-Whitney $U$ test (for continuous variables) and -Chi-square test (for categorical variables). Assumed predictors for adverse rebiopsy findings were examined by binary logistic regression analysis. Baseline predictors of time to treatment and time to adverse RP findings were analysed using Cox regression analysis. The Kaplan-Meier method was used to describe protocol-based treatment-free survival with respect to initial \% fPSA and \%fPSA velocity.

The ethics committee of each participating hospital approved the study protocol.

\section{Results}

Until October 2013, \%fPSA was measured from 939 out of 3701 PRIAS patients at the study enrolment. These 939 patients constitute the study cohort. \%fPSA was also available in 438 patients after 1 year of AS. Median follow-up was 17.2 months (range 0.7-82.7). By the time of analysis, $656(69.9 \%)$ patients of the study cohort were still on AS and 283 (30.1\%) had discontinued; of those, 181 (64.0\%) due to protocol-based reasons and $102(36.0 \%)$ because of other reasons such as anxiety, unknown reason or AS was changed to watchful waiting (WW). The median surveillance until discontinuation for all reasons was 14.7 months (range 0.7-82.7) and 14.9 months (range 4.0-54.8) for protocol-based reasons. Overall, $153(54.1 \%)$ patients discontinued AS and underwent surgical radical treatment (i.e. open retropubic/robot-assisted laparoscopic prostatectomy), $40(14.1 \%)$ had brachytherapy and $32(11.3 \%)$ had external beam radiotherapy. One $(0.4 \%)$ patient had HIFU 
Table 1 Characteristics of the patients enrolled in the study

\begin{tabular}{|c|c|c|c|c|}
\hline Variable & All patients $(n=939)$ & No treatment $(n=758)$ & Active treatment $(n=181)$ & $p$ value \\
\hline Age, year, median $(25-75 p)$ & $64.9(60.0-69.6)$ & $65.2(60.1-69.8)$ & $64.4(60.4-68.3)$ & 0.290 \\
\hline Prostate vol, $\mathrm{cm}^{3}$, median $(25-75 \mathrm{p})$ & $44.0(34.9-54.0)$ & $44.0(35.0-55.0)$ & $43.7(33.5-52.0)$ & 0.176 \\
\hline PSA, ng/mL, median (25-75p) & $5.6(4.5-7.0)$ & $5.6(4.5-6.8)$ & $5.8(4.8-7.3)$ & 0.063 \\
\hline \%fPSA, median (25-75p) & $14.5(9.7-18.8)$ & $14.7(10.1-19.7)$ & $13.5(8.6-18.6)$ & 0.068 \\
\hline PSA-D, ng/mL/g, median (25-75p) & $0.13(0.10-0.16)$ & $0.13(0.10-0.16)$ & $0.14(0.11-0.17)$ & 0.001 \\
\hline Clinical stage, no $(\%)$ & & & & 0.451 \\
\hline $\mathrm{T} 1 \mathrm{c}$ & $863(91.9)$ & $699(92.2)$ & $164(90.6)$ & \\
\hline $\mathrm{T} 2 \mathrm{a}-\mathrm{c}$ & $76(8.1)$ & $59(7.8)$ & $17(9.4)$ & \\
\hline Biopsy cores median, no (25-75p) & $12(10-12)$ & $12(10-12)$ & $12(10-12)$ & 0.004 \\
\hline Positive biopsy, no (\%) & & & & 0.050 \\
\hline 1 & $649(69.1)$ & $534(70.4)$ & $115(63.5)$ & \\
\hline 2 & $282(30.0)$ & $216(28.5)$ & $66(36.5)$ & \\
\hline NA & $8(0.9)$ & $8(1.1)$ & & \\
\hline
\end{tabular}

25-75p 25th and 75th percentiles, PSA prostate-specific antigen, $\%$ fPSA free/total PSA ratio (\%), PSA-D prostate-specific antigen density, NA not available

treatment, and in $19(6.7 \%)$ patients WW was initiated. A total of $38(13.4 \%)$ men had another treatment or the treatment chosen was not available.

Characteristics of the patients enrolled in the study are summarized in Table 1. First rebiopsy results were available for 595 of the study patients and in 144 (24.2\%) PC reclassification occurred. According to the multivariate binary logistic regression analysis, the predicting variables for adverse first rebiopsy findings were the number of PC positive cores (one vs. two) at the diagnostic biopsy $(p=0.000)$ and age at diagnosis $(p=0.002)$ (Table 2). PSA and \%fPSA could not predict PC reclassification.

In multivariate analysis, the initial \%fPSA value was not predictive for treatment change. After 1 year of AS, the difference in median \%fPSA values between patients still on surveillance $(16.6 \%)$ or discontinued $(13.1 \%)$ was statistically significant $(p=0.031)$. The probability to discontinue AS was significantly lower in patients with initial \%fPSA value $\geq 15$ and positive \%fPSA velocity compared to those with initial \%fPSA $<15$ and negative \%fPSA velocity $(p=0.000)$ (Fig. 1). Cox regression analysis could not demonstrate an association between \%fPSA and treatment change over time, but did show this for PSA-D and the number of PC positive cores at the diagnostic biopsy (Table 3). According to Cox regression analysis, PSA-D ( $p=0.028$ ) was the only predictive baseline variable for unfavourable findings at RP (data not shown).

\section{Discussion}

The aim of the study was to evaluate the hypothesis that \%fPSA has a role as a prognostic tool in AS. More
Table 2 Association of baseline characteristics with rebiopsy reclassification

\begin{tabular}{lll}
\hline Baseline characteristics & $\begin{array}{l}\text { 1-year rebiopsy }(n=595) \text { OR } \\
(95 \% \mathrm{Cl})\end{array}$ & $p$ value \\
\hline Age at dg & $1.1(1.02-1.09)$ & $0.002 *$ \\
PSA & $0.96(0.74-1.25)$ & 0.79 \\
\%fPSA & $1.0(0.99-1.04)$ & 0.41 \\
PSA-D & $1.1(0.95-1.22)$ & 0.26 \\
Clinical stage & & 0.17 \\
T1c & Ref. & \\
T2 & $1.62(0.81-3.24)$ & 0.64 \\
Total Bx cores & $0.98(0.88-1.08)$ & $0.000^{*}$ \\
Positive Bx cores & & \\
1 & Ref. & \\
2 & $2.06(1.38-3.09)$ & \\
\hline
\end{tabular}

$P S A$ prostate-specific antigen, \% $P P S A$ free/total PSA ratio (\%), PSA$D$ prostate-specific antigen density, $O R$ odds ratio, $C I$ confidence interval

* Significant result $(p<0.05)$

specific, we wanted to address whether the \%fPSA value at diagnosis or during follow-up associates with adverse rebiopsy or RP findings and whether treatment change can be predicted. Our results show that, although \%fPSA values were statistically significantly different in the groups analysed (still on AS versus discontinued), no clinically relevant correlations of diagnostic \%fPSA were evident to guide clinical practice. Interestingly, however, the probability of continuing AS was the highest in patients with favourable \%fPSA characteristics at diagnosis and during follow-up. 


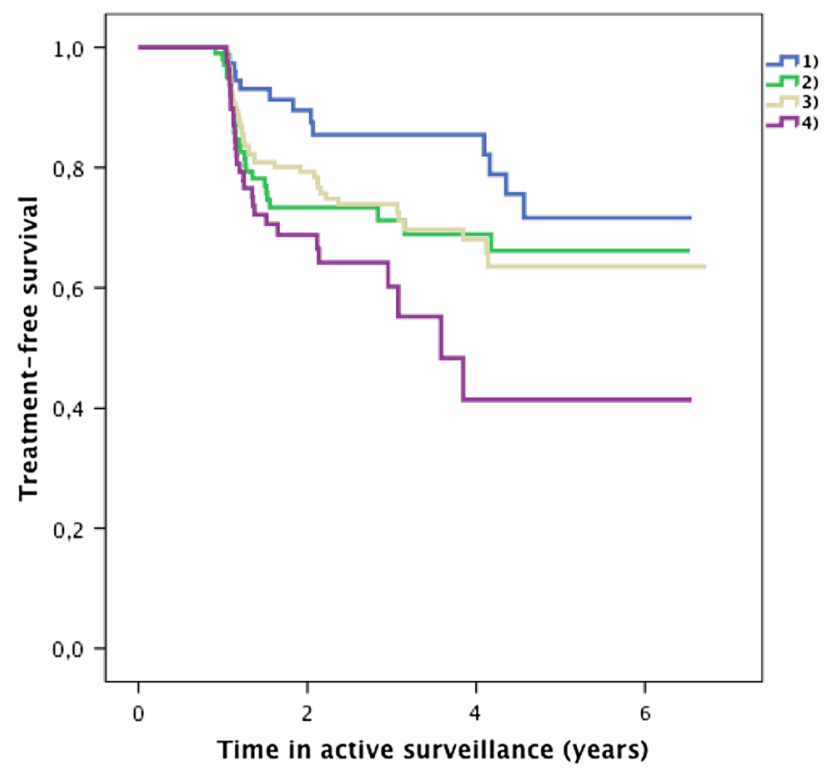

Fig. 1 Treatment-free survival of study patients stratified into subgroups based on \%fPSA characteristics. $1 \%$ fPSA $\geq 15$ and $\%$ fPSA velocity positive (blue), $2 \%$ fPSA $\geq 15$ and $\%$ fPSA velocity negative (green), $3 \%$ fPSA $<15$ and \%fPSA velocity positive (yellow), 4 $\%$ fPSA $<15$ and \%fPSA velocity negative (purple), statistical differences between groups: $p<0.05,1$ versus $4 ; p \geq 0.05$, all other comparisons

Table 3 Cox regression analysis of baseline characteristics with treatment change (protocol-based) over time

\begin{tabular}{lll}
\hline Baseline characteristics & $\begin{array}{l}\text { Treatment change }(n=181) \mathrm{HR} \\
(95 \% \mathrm{Cl})\end{array}$ & $p$ value \\
\hline Age at dg & $1.0(0.97-1.02)$ & 0.81 \\
PSA & $1.0(0.94-1.14)$ & 0.48 \\
\%fPSA & $0.99(0.88-1.20)$ & 0.74 \\
PSA-D & $1.1(1.01-1.11)$ & $0.013^{*}$ \\
Clinical stage & & 0.39 \\
T1c & Ref. & \\
T2 & $1.3(0.74-2.18)$ & 0.92 \\
Total Bx cores & $1.0(0.92-1.08)$ & $0.019^{*}$ \\
Positive Bx cores & & \\
1 & Ref. & \\
2 & $1.5(1.07-2.01)$ & \\
\hline
\end{tabular}

PSA prostate-specific antigen, $f P S A \%$ free/total PSA ratio (\%), PSA$D$ prostate-specific antigen density, $H R$ hazard ratio

* Significant result $(p<0.05)$

While the strengths of our study are its prospective nature and the relatively large patient cohort, obvious shortcomings do exist. \%fPSA was not routinely used, and thus only $25 \%$ of the patients entering the entire PRIAS trial were available for the study and a bias can not be excluded. Also, short follow-up and lack of randomization comparing radical treatments of PC with AS make generalization of the results difficult.

\%fPSA has been widely studied as a marker to predict the probability of PC diagnosis $[11,12]$. The \%fPSA has been used to stratify the risk of PC in men with the total PSA value between 4 and $10 \mathrm{ng} / \mathrm{mL}$ and a negative DRE finding. However, after PC diagnosis or PSA being more than $10 \mathrm{ng} / \mathrm{mL}$, \%fPSA has not been of clinical use. The association between low \% fPSA and high-grade PC with aggressive features has been demonstrated previously [16, 17], as well as the association between \%fPSA and clinically significant PC, based on analyses in a multivariate setting [18]. However, only a few studies have addressed the role of \% fPSA as a prognostic tool in AS. Contradicting our findings, previous studies have concluded that \%fPSA predicts time to deferred radical treatment [5, 19] or adverse rebiopsy results [19] in patients with lowrisk PC managed by AS. One explanation may be that PRIAS patients represent a relatively homogeneous patient cohort. In PRIAS, only minimal Gleason score 6 cancers are allowed as opposed to other trials where Gleason 7 $(3+4)$ cancers were also included, which likely increases the power of the analysis. Also, the used \%fPSA cut-off, the definition of adverse rebiopsy findings and length of follow-up were all different hampering the comparability of the results. Importantly, only first rebiopsy results were used for the analysis in our study due to a limited number of patients with both \%fPSA and later rebiopsy data available. It is likely that a change in Gleason score at rebiopsy at one year represent initial diagnostic misclassification instead of true disease progression. Therefore, longer follow-up is needed to clarify the role of diagnostic \%fPSA in AS. In multivariate analysis, PSA-D and the number of PC positive cores were the baseline characteristics that associated with treatment change. This is in concordance with the data from other AS cohorts $[20,21]$ and a recent PRIAS analysis [3] thus supporting the validity of the current analysis.

Although baseline \%fPSA could not predict treatment change, the median \%fPSA at 1 year was significantly higher in patients able to continue AS than in patients that discontinued. The difference between the groups was particularly clear, if initial \%fPSA and \%fPSA velocity were used as a joined categorical variable. The probability of continuing AS was highest in men with favourable \%fPSA initially and during follow-up. Literature on the utility of \%fPSA velocity in AS is scarce. Although scepticism over PSA kinetics in general has been expressed [22], to our knowledge, \%fPSA velocity has not been explored as a predictor of AS outcome before in the literature. However, a recent congress abstract supports the findings in our study [Heidegger I et al., EAU Milan, 2013]. Perhaps longitudinal biomarker measurements (e.g. \%fPSA-kinetics) may 
have better predictive accuracy than a single baseline measurement in AS programs [23]. Also serial biomarker measurements over time provide greater potential to characterize the tumour more accurately than on the basis of a single baseline value. This is especially interesting in the light of recently published data suggesting that PSA velocity risk count (expressed as the number of times PSA velocity exceeds a certain threshold in a certain time period) is independently associated with adverse rebiopsy findings in an AS cohort [24]. In our study, the baseline variables' predictive of adverse rebiopsy results was the number of positive biopsy cores and age, which is in line with the results from other AS series [3-7].

Our results showed no association between \%fPSA and RP findings. The only predictive baseline variable was PSA-D, which has also been shown earlier [25]. Contrary to our findings, previous studies have demonstrated the predictive capability of \%fPSA for significant cancer in RP. A low \%fPSA has been associated with significant upgrading of low-grade PC after RP [13], adverse pathological features following surgery [26] and higher stage in RP [27, 28]. It is likely that the power needed for analyses is not reached in our study due to the small number of RPs done. Therefore longer follow-up and larger series are needed to clarify this issue.

In conclusion, diagnostic \%fPSA alone provided no additional prognostic benefit when compared to other predictors used in AS protocols, such as PSA kinetics. However, \%fPSA coupled to \%fPSA velocity during may increase the ability to predict patients at risk to change treatment during AS.

Acknowledgments This study was supported by Grant from the Biomedicum Helsinki Foundation and the Finnish Cancer Society.

Conflict of interest The authors declare that they have no conflict of interest.

\section{References}

1. Cooperberg MR, Broering JM, Kantoff PW, Carroll PR (2007) Contemporary trends in low risk prostate cancer: risk assessment and treatment. J Urol 178(3 Pt 2):S14-S19

2. Parker C (2004) Active surveillance: towards a new paradigm in the management of early prostate cancer. Lancet Oncol 5(2):101-106

3. Bul M, Zhu X, Valdagni $R$ et al (2013) Active surveillance for low-risk prostate cancer worldwide: the PRIAS study. Eur Urol 63(4):597-603

4. Dall'Era MA, Konety BR, Cowan JE et al (2008) Active surveillance for the management of prostate cancer in a contemporary cohort. Cancer 112(12):2664-2670

5. van As NJ, Norman AR, Thomas K et al (2008) Predicting the probability of deferred radical treatment for localised prostate cancer managed by active surveillance. Eur Urol 54(6):1297-1305
6. Klotz L, Zhang L, Lam A, Nam R, Mamedov A, Loblaw A (2010) Clinical results of long-term follow-up of a large, active surveillance cohort with localized prostate cancer. J Clin Oncol 28(1):126-131

7. Tosoian JJ, Trock BJ, Landis P et al (2011) Active surveillance program for prostate cancer: an update of the Johns Hopkins experience. J Clin Oncol 29(16):2185-2190

8. Ouzzane A, Puech P, Villers A (2012) MRI and surveillance. Curr Opin Urol 22(3):231-236

9. Stamatakis L, Siddiqui MM, Nix JW et al (2013) Accuracy of multiparametric magnetic resonance imaging in confirming eligibility for active surveillance for men with prostate cancer. Cancer 119(18):3359-3366

10. Isharwal S, Makarov DV, Sokoll LJ et al (2011) ProPSA and diagnostic biopsy tissue DNA content combination improves accuracy to predict need for prostate cancer treatment among men enrolled in an active surveillance program. Urology 77(3):763.e1-763.e6

11. Catalona WJ, Smith DS, Wolfert RL et al (1995) Evaluation of percentage of free serum prostate-specific antigen to improve specificity of prostate cancer screening. JAMA 274(15):1214-1220

12. Partin AW, Catalona WJ, Southwick PC, Subong EN, Gasior GH, Chan DW (1996) Analysis of percent free prostate-specific antigen (PSA) for prostate cancer detection: influence of total PSA, prostate volume, and age. Urology 48(6A Suppl):55-61

13. Visapaa H, Hotakainen K, Lundin J, Ala-Opas M, Stenman UH (2010) The proportion of free PSA and upgrading of biopsy Gleason score after radical prostatectomy. Urol Int 84(4):378-381

14. Ramirez ML, Nelson EC, Deverewhite RW, Lara PN Jr, Evans CP (2008) Current applications for prostate-specific antigen doubling time. Eur Urol 54(2):291-300

15. van den Bergh RC, Roemeling S, Roobol MJ, Roobol W, Schroder FH, Bangma CH (2007) Prospective validation of active surveillance in prostate cancer: the PRIAS study. Eur Urol 52(6):1560-1563

16. Southwick PC, Catalona WJ, Partin AW et al (1999) Prediction of post-radical prostatectomy pathological outcome for stage T1c prostate cancer with percent free prostate specific antigen: a prospective multicenter clinical trial. J Urol 162(4):1346-1351

17. Catalona WJ, Southwick PC, Slawin KM et al (2000) Comparison of percent free PSA, PSA density, and age-specific PSA cutoffs for prostate cancer detection and staging. Urology 56(2):255-260

18. Nam RK, Toi A, Klotz LH et al (2007) Assessing individual risk for prostate cancer. J Clin Oncol 25(24):3582-3588

19. Selvadurai ED, Singhera M, Thomas K et al (2013) Mediumterm outcomes of active surveillance for localised prostate cancer. Eur Urol 64(6):981-987

20. San Francisco IF, Werner L, Regan MM, Garnick MB, Bubley G, DeWolf WC (2011) Risk stratification and validation of prostate specific antigen density as independent predictor of progression in men with low risk prostate cancer during active surveillance. J Urol 185(2):471-476

21. Kotb AF, Tanguay S, Luz MA, Kassouf W, Aprikian AG (2011) Relationship between initial PSA density with future PSA kinetics and repeat biopsies in men with prostate cancer on active surveillance. Prostate Cancer Prostatic Dis 14(1):53-57

22. Iremashvili V, Manoharan M, Lokeshwar SD, Rosenberg DL, Pan D, Soloway MS (2013) Comprehensive analysis of postdiagnostic prostate-specific antigen kinetics as predictor of a prostate cancer progression in active surveillance patients. BJU Int 111(3):396-403

23. Ng MK, Van As N, Thomas K et al (2009) Prostate-specific antigen (PSA) kinetics in untreated, localized prostate cancer: PSA velocity versus PSA doubling time. BJU Int 103(7):872-876 
24. Patel HD, Feng Z, Landis P, Trock BJ, Epstein JI, Carter HB (2013) Prostate specific antigen velocity risk count predicts biopsy reclassification for men with very low risk prostate cancer. J Urol 191(3):629-637

25. Vellekoop A, Loeb S, Folkvaljon Y, Stattin P (2014) Population based study of predictors of adverse pathology among candidates for active surveillance with gleason 6 prostate cancer. J Urol 191(2):350-357

26. Steuber T, Vickers AJ, Serio AM et al (2007) Comparison of free and total forms of serum human kallikrein 2 and prostate-specific antigen for prediction of locally advanced and recurrent prostate cancer. Clin Chem 53(2):233-240

27. Southwick PC, Catalona WJ, Partin AW et al (1999) Prediction of post-radical prostatectomy pathological outcome for stage T1c prostate cancer with percent free prostate specific antigen: a prospective multicenter clinical trial. J Urol 162(4):1346-1351

28. Elabbady AA, Khedr MM (2006) Free/total PSA ratio can help in the prediction of high gleason score prostate cancer in men with total serum prostate specific antigen (PSA) of 3-10 ng/ml. Int Urol Nephrol 38(3-4):553-557 